\title{
TRITERPENOS ISOLADOS DE ESCHWEILERA LONGIPES MIERS (LECYTHIDACEAE)
}

Mario Geraldo de Carvalho, Javier Rincón Velandia, Lucilene Faustina de Oliveira e Flavio Barbosa Bezerra Departamento de Química - Instituto de Ciências Exatas - Universidade Federal Rural do Rio de Janeiro - 23851-970 - Seropédica Itaguaí - RJ

Recebido em 15/12/97; aceito em 26/1/98

\begin{abstract}
TRITERPENES ISOLATED FROM ESCHWEILERA LONGIPES MIERS (LECYTHIDACEAE). The phytochemical studies of Eschweilera longipes have led to the identification of ten triterpenoids: fridelin, fridelinol, $\alpha$-amirin, $\beta$-amirin, 3 $\beta$-O-cinamoyl- $\alpha$-amirin, $3 \beta$-O-cinamoyl- $\beta$-amirin, $\alpha$-amirenone, $\beta$-amirenone, 3- $\alpha$-hidroxi-lupeol, 3- $\alpha$-hidroxi-taraxasterol, along with $\beta$-sitosterol, stigmasterol, $\alpha$-tocopherol and tocotrienol. The structures of these compounds were identified by analysis of $I R,{ }^{1} \mathrm{H}$ and ${ }^{13} \mathrm{C}$ NMR data and comparison with values of literature.
\end{abstract}

Keywords: Eschweilera longipes; Lecythidaceae; triterpenes.

\section{INTRODUÇÃO}

A família Lecythidaceae é constituída de 25 gêneros e 400 espécies que ocorrem na forma de árvores, com uma distribuição pantropical ${ }^{1}$.

Alguns constituintes com atividade farmacológica têm sido isolados de espécies desta família e, por isso, devem-se desenvolver estudos fitoquímicos e farmacológicos de espécies de Lecythidaceae. Os trabalhos relacionados com estudo químico de espécies desta família conduziram a identificação de triterpenos pentacíclicos, saponinas, ácido elágico e alcalóides do tipo indolo[2,1-b]quinazolínicos ${ }^{2-6}$.

Espécies do gênero Eschweilera ocorrem frequentemente no norte e nordeste do Brasil e são usadas na indústria madeireira, em obras, em cercas e como combustível doméstico. Além das comunicações sobre estudo químico de . $_{\text {ovata }}{ }^{7 \mathrm{a}}$, E. rabeliana $^{7 \mathrm{~b}}$ $e$ E. longipes $^{7 \mathrm{c}}$, há apenas uma publicação relacionada com a química deste gênero que revela a presença de triterpenos pentacíclicos nas cascas e folhas de E. rabeliana ${ }^{8}$. O presente trabalho vem ampliar o conhecimento sobre a composição química deste gênero e portanto da família Lecythidaceae.

\section{RESULTADOS E DISCUSSÃO}

Fracionamento cromatográfico e purificação por cristalização de frações dos extratos das cascas e folhas de E. longipes conduziram ao isolamento e a identificação de quatorze terpenóides. As substâncias 3, 4, 7-14 estão sendo identificadas pela primeira vez neste gênero.

A identificação das substâncias isoladas foi feita através da análise dos espectros de IV, RMN ${ }^{1} \mathrm{H}$ e RMN ${ }^{13} \mathrm{C}$ envolvendo comparação com dados da literatura. A feição dos espectros de RMN ${ }^{1} \mathrm{H}$ permitiu identificar três classes distintas das substâncias: 1-8, 13 e 14 (triterpenos pentacíclicos), 9 e 10 (esteróides) e dois cromanóis (11-12). A comparação dos espectros de RMN ${ }^{13} \mathrm{C}$ totalmente desacoplado (PND) e RMN ${ }^{13} \mathrm{C}$-DEPT $\left(\theta=90^{\circ} \mathrm{e}\right.$ $\left.\theta=135^{\circ}\right)$ permitiu identificar o número de carbonos metílicos, metilênicos e metínicos. Os sinais restantes do espectro PND foram identificados como representantes dos carbonos quaternários. Com base nesses dados obtidos, na aplicação da metodologia de Olea e col. ${ }^{9}$ e na correlação dos valores das frequências dos sinais de alguns carbonos funcionalizados (carbonos carbonílicos, metínicos carbinólicos e olefínicos) foi possível classificar os triterpenos 1 e $\mathbf{2}$ na série fridelano, $\mathbf{3}, \mathbf{5}$ e $\mathbf{7}$ na série urseno e 4, 6 e 8 na série oleaneno ${ }^{11,12}$.

O triterpeno 1 apresentou sinal de estiramento de carbonila em
$1715 \mathrm{~cm}^{-1}\left(v_{\mathrm{C}=\mathrm{O}}\right)$ no espectro IV. O espectro de RMN ${ }^{13} \mathrm{C}$ apresentou sinal de carbono carbonílico em 214,50 ppm e um sinal de um grupo metila em $6,40 \mathrm{ppm}$ protegido pelo efeito $\gamma$ do oxigênio da carbonila. $\mathrm{O}$ triterpeno 2 apresentou absorção em $3500 \mathrm{~cm}^{-1}$ $\left(v_{\mathrm{O}-\mathrm{H}}\right)$ e $1100\left(v_{\mathrm{C}-\mathrm{O}}\right)$ no espectro IV. O espectro de RMN ${ }^{13} \mathrm{C}$ mostrou, entre outros, um sinal em 72,10 ppm, que corresponde ao deslocamento químico de um carbono carbinólico, compatível com o sinal em 3,70 ppm (m) revelado no espectro de RMN ${ }^{1} \mathrm{H}$. A comparação dos valores de deslocamentos químicos dos carbonos de 1 e 2 (Tabela-1) com valores registrados na literatura para a fridelina e o fridelinol ${ }^{10-14}$ permitiu identificar estes triterpenos como 3-oxo-fridelano (fridelina) e 3 $\beta$-hidroxi-fridelano (fridelinol).

$\mathrm{O}$ espectro no IV da mistura de $\mathbf{3}$ e $\mathbf{4}$ apresentou as bandas de carbonila de éster conjugado $\left(1725 \mathrm{~cm}^{-1}\right)$ e do sistema aromático (1600 e $1500 \mathrm{~cm}^{-1}$ ). O espectro de $\mathrm{RMN}{ }^{1} \mathrm{H}$ apresentou dois dubletos em 6,44 e 7,65 ppm $(\mathrm{J}=16,0 \mathrm{~Hz})$ e dois multipletos em 7,37 ppm $(3 \mathrm{H})$ e 7,52 ppm $(2 \mathrm{H})$. Estes dados estão compatíveis com a unidade cinamoila que foi confirmada pelos valores de deslocamentos químicos no espectro de $\mathrm{RMN}{ }^{13} \mathrm{C}\left(\delta_{\mathrm{C}}: 166,70 \mathrm{e}\right.$ 134,$40 ; \delta_{\mathrm{CH}}: 144,20,130,00,128,70,127,90$ e $\left.118,70 \mathrm{ppm}\right) . \mathrm{O}$ multipleto em 4,65 ppm presente no espectro de $\mathrm{RMN}{ }^{1} \mathrm{H}$ e o sinal em $80,60 \mathrm{ppm}$ no espectro de $\mathrm{RMN}{ }^{13} \mathrm{C}$ foram atribuídos ao CH-3 da unidade terpênica ligada ao grupo cinamoila. Os sinais observados no espectro de $\mathrm{RMN}{ }^{13} \mathrm{C}$ na região de carbono $\mathrm{sp}^{2}$ $\left(\delta_{\mathrm{C}}: 146,00\right.$ e $140,00 \mathrm{ppm} ; \delta_{\mathrm{CH}}: 124,20$ e $\left.122,20 \mathrm{ppm}\right)$ permitiram classificá-los nas séries: urseno $\left(\mathrm{C}-13: \delta_{\mathrm{C}} 140,00 \mathrm{ppm}\right.$; C-12: $\delta_{\mathrm{CH}}$ $124,20 \mathrm{ppm}$ ), que aparece em maior percentagem, e oleaneno (C13: $\left.\delta_{\mathrm{C}} 146,00 \mathrm{ppm} ; \mathrm{C}-12: \delta_{\mathrm{CH}} 122,20 \mathrm{ppm}\right)^{2,3,9-13}$. Os sinais em $5,13 \mathrm{ppm}$ e $5,15 \mathrm{ppm}$ presentes no espectro de RMN ${ }^{1} \mathrm{H}$ correspondem aos hidrogênios olefínicos em C-12. Após esta análise, compararam-se os dados registrados na literatura para a $\alpha$ amirina $^{11,15-17}$ e para o cinamato de $\beta$-amirina $(4)^{18,19}$. Esta comparação permitiu identificar os constituintes da mistura como cinamato de $\alpha$-amirina (3) e cinamato de $\beta$-amirina (4) (Tabela- 1 ). Os deslocamentos químicos dos carbonos de $\mathbf{3}$ não foram encontrados na literatura $^{11,20}$.

A mistura de 5 e 6 apresentou absorções de $v_{\mathrm{OH}}\left(3500 \mathrm{~cm}^{-1}\right)$ e $v_{\mathrm{C}=\mathrm{C}}\left(1657 \mathrm{~cm}^{-1}\right)$ no espectro IV. O espectro de RMN ${ }^{1} \mathrm{H}$ desta mistura apresentou sinais de prótons olefínicos $(5,10 \mathrm{ppm}$; $\mathrm{m})$, de prótons carbinólicos $(3,20$ ppm; dd; $J=10,0$ e $6,0 \mathrm{~Hz})$ e a feição dos sinais entre 1,05 e $0,77 \mathrm{ppm}$ foi semelhante à da mistura de 3 e 4. Os sinais dos carbonos olefínicos (C-12 e C-13) no espectro de $\mathrm{RMN}{ }^{13} \mathrm{C}(\delta 145,20 ; 139,60 ; 124,40$ e $121,60 \mathrm{ppm})$, de carbono carbinólico (C-3, 79,60 ppm) e as observações citadas acima permitiram identificar as estruturas da $\alpha$ - e $\beta$-amirina para as substâncias 5 e 6. Os deslocamentos químicos dos 
demais carbonos foram semelhantes aos dados de $\mathbf{3 + 4}$ e aos valores registrados na literatura ${ }^{11,16-18}$ para as substâncias não aciladas (Tabela-1).

Os triterpenos $\mathbf{7}$ e $\mathbf{8}$ foram identificados como constituintes de uma mistura com base nos dados de $\mathrm{RMN}{ }^{1} \mathrm{H}[5,10 \mathrm{ppm}$ (m), 2,50-2,20 ppm (m) e sinais de grupos metílicos entre 1,11$0,76 \mathrm{ppm}]$. A principal diferença observada na comparação dos espectros de $\mathrm{RMN}{ }^{13} \mathrm{C}$ de $\mathbf{7 + 8}$ e $\mathbf{5 + 6}$ deve-se à presença do sinal em $\delta_{\mathrm{C}} 217,90 \mathrm{ppm}(\mathbf{7 + 8})$ e na ausência do sinal 79,60 ppm. A maior intensidade do par de sinais $\delta_{C} 139,70 / 124,20$ sugere uma maior percentagem de 7 onde o C-13 encontra-se protegido pelo efeito $\gamma$ exercido pelo grupo metílico ligado ao C-19. A comparação dos deslocamentos químicos dos carbonos das substâncias desta mistura com os valores descritos na literatura para $\alpha$ - e $\beta$-amirenona ${ }^{9,11,21,22}$ e com os dados obtidos para os triterpenos 5 e 6 (Tabela-1) permitiram definir as estruturas de $\mathbf{7 + 8}$ como 3-oxo-ursa-12(13)-eno (7) e 3-oxoolean-12(13)-eno $(\mathbf{8})^{22}$. Trabalhos publicados em revista nacional registraram os dados de $\mathrm{RMN}{ }^{13} \mathrm{C}$ destas substâncias. Entretando estes dados não são citados nas revisões publicadas recentemente sobre triterpenos pentacíclicos ${ }^{11}$ e não constam, também, no dicionário de produtos naturais ${ }^{20}$.

A mistura dos esteróides $\mathbf{9}$ e $\mathbf{1 0}$ foi identificada através dos sinais presentes no espectro de $\mathrm{RMN}{ }^{1} \mathrm{H}$ em $\delta_{\mathrm{H}} 0,62(\mathrm{~s}), 1,05$ (s), 0,74-0,98 (m) correspondentes a absorção de grupos metílicos de esteróides, de um multipleto em $\delta_{\mathrm{H}} 3,49 \mathrm{ppm}(\mathrm{H}-$ 3) e dos sinais em $\delta_{\mathrm{H}} 5,10(\mathrm{~m}, \mathrm{H}-22$ e $\mathrm{H}-23,10)$ e $\delta_{\mathrm{H}} 5,50[\mathrm{dl}$, H-6 $(\mathbf{9 + 1 0})$ de prótons olefínicos. Estes dados, aliados à análise do espectro de $\mathrm{RMN}{ }^{13} \mathrm{C}$ da mistura e comparação com dados da literatura ${ }^{23}$, permitiram identificar as estruturas do $\beta$ sitosterol (9) e do estigmasterol (10). A percentagem relativa (1:1) entre os componentes na mistura foi deduzida através da análise da integração dos sinais dos prótons olefínicos, da mesma forma divulgada na literatura ${ }^{23}$.

Os espectro de RMN ${ }^{1} \mathrm{H}$ das frações contendo a mistura de $\mathbf{1 1}$ e 12 apresentou os sinais em 0,83 ppm (d, 6,4 Hz), 1,22ppm (s), 1,96-2,2 ppm (m) e 2,2 ppm (s). Estes sinais são semelhantes aos espectros do $\alpha$-tocoferol $(\mathbf{1 1})^{24}$. Os espectros de RMN ${ }^{13} \mathrm{C}$ (PND e DEPT) apresentaram sinais de carbonos metílicos $\left(\delta_{\mathrm{CH} 3}: 11,7\right.$; 11,$8 ; 12,0 ; 19,2 ; 22,7 ; 22,7$ e 23,6 ppm), carbonos metilênicos $\left(\delta_{\mathrm{CH} 2}: 41,4 ; 39,3 ; 37,4 ; 31,4 ; 24,8 ; 24,5 ; 22,6 ; 21,0\right.$ e 21,0 ppm$)$, metínicos $\left(\delta_{\mathrm{CH}}: 27,9,32,7,32,8 \mathrm{ppm}\right)$ e carbonos quaternários $\left(\delta_{\mathrm{C}}: 145,2 ; 144,4 ; 122,4 ; 122,2 ; 121,8 ; 115,7 ; 115,3 ; 114,9\right.$ e 74,6 ppm) semelhantes ao espectro da amostra autêntica da vitamina E e aos valores da literatura ${ }^{25}$. Os demais sinais presentes no espectro de $\mathrm{RMN}{ }^{1} \mathrm{H}$ como os de grupos metílicos ligados aos carbonos $\mathrm{sp}^{2}(1,56 ; 1,58 ; 1,66 ; \mathrm{s})$, de prótons olefínicos $[5,30$ e 5,07 (m)] em conjunto com outros sinais de carbonos $\mathrm{sp}^{2}$-metínicos $(124,0$; $124,1)$ e quaternários $(135,2 ; 131,0)$ no espectro de $\mathrm{RMN}{ }^{13} \mathrm{C}$ (PND e DEPT) (Tabela-1) estão de acordo com os dados espectrométricos do tocotrienol $(\mathbf{1 2})^{25,26}$.

Os triterpenos 13 e 14 foram identificados através da análise dos espectros de RMN ${ }^{1} \mathrm{H}$ e ${ }^{13} \mathrm{C}(\mathrm{PND}$ e DEPT) da mistura contendo apenas os dois componentes. Os sinais presentes no espectro de $\mathrm{RMN}{ }^{1} \mathrm{H}$ em 3,70 ppm(sl), 4,56(s) e 4,65(s) e 1,67 ppm(s) foram atribuídos, respectivamente, aos prótons $\mathrm{H}-3, \mathrm{H}-29$ a e $\mathrm{H}-$ $29 \mathrm{~b}$ e H-30 do lupeol e os sinais em 3,70 (sl), 4,35 (s) e 4,40 ppm (s) foram atribuídos, respectivamente, aos prótons $\mathrm{H}-3, \mathrm{H}-29 \mathrm{a}$ e $\mathrm{H}-29 \mathrm{~b}$ do taraxasterol. Os sinais presentes no espectro de RMN ${ }^{13} \mathrm{C}\left[\delta_{\mathrm{CH} 2}: 109,1(\mathbf{1 3}), 106,1(\mathbf{1 4}) ; \delta_{\mathrm{C}}: 153,1(\mathbf{1 3})\right.$ e $156,0(\mathbf{1 4})$; $\delta_{\mathrm{CH}}: 75,0(\mathbf{1 3}$ e 14)] e os demais sinais foram comparados com os valores registrados na literatura para o lupeol, taraxasterol e derivados destes triterpenos com configuração 3 - $\alpha$-hidroxi ${ }^{11,14}$. Esta comparação permitiu verificar que o valor 75,0 ppm é compatível com grupo HO-3 em alfa exercendo efeito $\gamma$ de proteção sobre os carbonos C-1 e C-5. Estas informações permitiram propor a estrutura do 3- $\alpha$-hidroxi-lupeol para 13 e 3 - $\alpha$-hidroxi-taraxasterol para 14 cujos deslocamentos químicos dos átomos de carbono-13 estão sendo registrados pela primeira vez na literatura (Tabela-1).
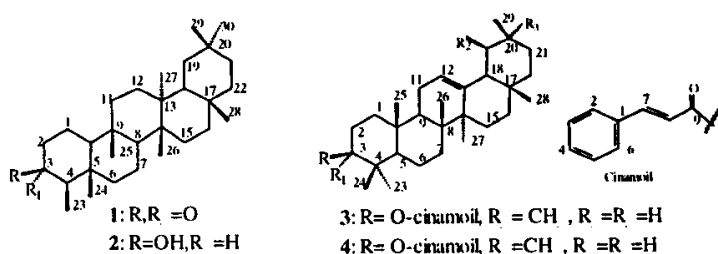

3: $\mathrm{R}=\mathrm{O}$-ciumosil, $\mathrm{R}=\mathrm{CH}, \mathrm{R}=\mathrm{R}=\mathrm{H}$ 4: $\mathrm{R}=\mathrm{O}$-cintmoil, $\mathrm{R}=\mathrm{CH}, \mathrm{R}=\mathrm{R}=\mathrm{H}$ 5: $\mathrm{R}=\mathrm{OH}, \mathrm{R}_{2}=\mathrm{CH}_{3}, \mathrm{R}_{1}=\mathrm{R}_{3}=\mathrm{H}$ 6: $\mathrm{R}=\mathrm{OH}, \mathrm{R}_{1}=\mathrm{R}_{2}=\mathrm{H}, \mathrm{R}_{3}=\mathrm{CH}_{3}$ 7: $\mathrm{R}, \mathrm{R}_{1}=\mathrm{O}, \mathrm{R}_{2}=\mathrm{CH}_{3}, \mathrm{R}_{3}=\mathrm{H}$

$8: R_{1} R_{1}=O, R_{2}=H . R_{3}=C_{3}$
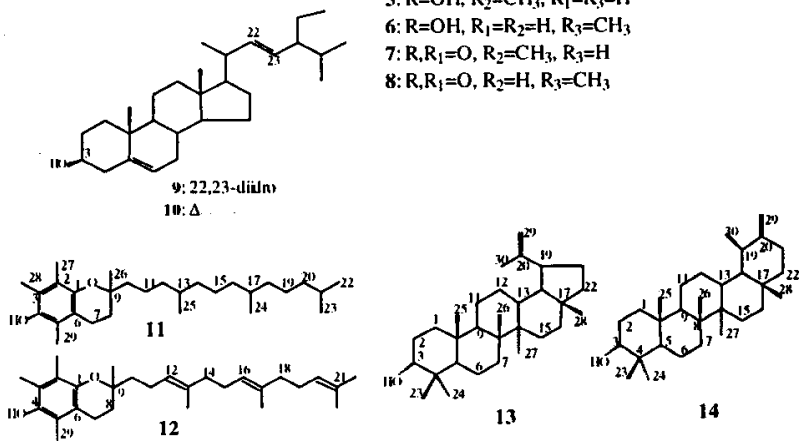

\section{PARTE EXPERIMENTAL}

Procedimentos experimentais gerais. Os pontos de fusão (PF) foram deteminados em aparelho Kofler e não foram corrigidos. Os espectros na região do IV foram obtidos em um espectrômetro Perkin-Elmer modelo 1420, em pastilha de $\mathrm{KBr}$. Os espectros de RMN foram registrados em instrumento AC$200\left({ }^{1} \mathrm{H}\right.$ : $200.13 \mathrm{MHz}$ e $\left.{ }^{13} \mathrm{C}: 50.3 \mathrm{MHz}\right)$ da Bruker, usando-se $\mathrm{CDCl}_{3}$ como solvente e TMS como referência interna. Nas separações cromatográficas em coluna e camada fina analítica e preparativa usou-se gel de sílica da Aldrich ou Merck com granulação adequada. As placas cromatográficas foram reveladas com luz UV (254 $\mathrm{nm}$ ) e vapores de iodo.

Material vegetal. O espécimen de E. longipes utilizado neste estudo foi coletado no estado do Amapá, no mês de maio de 1991, e foi identificado pelo botânico Benedito Vitor Rabelo. Uma excicata ( $\mathrm{n}^{\mathrm{o}}$ 00358) encontra-se arquivada no Herbário Amapaense do Museu Angelo Moreira da Costa Lima-IEPA, Macapá, Amapá, Brasil.

Extração e isolamento dos constituintes químicos. As cascas $(1,2 \mathrm{~kg})$ e folhas $(0,7 \mathrm{~kg})$ da planta foram secas, moídas e submetidas a extração com solventes orgânicos através de maceração a frio. As soluções foram concentradas em evaporador rotativo sob vácuo. Obtiveram-se os resíduos dos extratos hexânicos das cascas (ELCH: 8,8 g) e das folhas (ELFH: 2,1 g). $\mathrm{O}$ extrato ELCH foi cristalizado em hexano e uma porção do material sólido foi separada por filtração sob vácuo e fracionada através de cromatografia em camada preparativa centrífuga (chromatotron) usando hexano/acetato de etila $(8: 2)$ como eluente. Por este procedimento obtiveram-se 1 (92,0 mg), $2(65,0 \mathrm{mg})$ e uma mistura de $\mathbf{3 + 4}(150,0 \mathrm{mg})$. As frações intermediárias eram constituídas de mistura destes componentes. A água mãe foi concentrada sob vácuo e o resíduo foi fracionado em coluna de sílica gel, usando diclorometano como eluente inicial e alterando-se a polaridade com metanol até metanol puro. Recolheram-se desta coluna 307 frações de $5 \mathrm{ml}$ cada uma que foram analisadas através de cromatografia em camada fina e reunidas em grupos de frações. As primeiras frações eram constituídas de um material oleoso, amarelo alaranjado, cuja análise por $\mathrm{RMN}{ }^{1} \mathrm{H}$ e ${ }^{13} \mathrm{C}$ revelou uma mistura de $\mathbf{1 1}+\mathbf{1 2}(50,0 \mathrm{mg})$ e as seguintes eram constituídas deste óleo e material cristalino. As frações que continham maior quantidade de material foram recristalizadas em metanol e o produto cristalino foi filtrado em gel de sílica usando diclorometano/acetato de etila (9:1) como eluente fornecendo, além de uma mistura de ésteres de triterpenos, a mistura de $\beta$-sitosterol e estigmasterol (9+10; PF: $\left.134-36^{\circ} \mathrm{C} ; 53,0 \mathrm{mg}\right)$. As 
Tabela 1. Dados de $\mathrm{RMN}{ }^{13} \mathrm{C}(50,3 \mathrm{MHz})$ das substâncias 1-8, 11-14 $\left(\mathrm{CDCl}_{3} \text {, TMS, } \delta\right)^{\mathrm{a}}$

\begin{tabular}{|c|c|c|c|c|c|c|c|c|c|c|c|c|}
\hline$C$ & 1 & 2 & $3^{b}$ & $4^{b}$ & 5 & 6 & 7 & 8 & $11^{d}$ & $12^{d}$ & 13 & 14 \\
\hline 1 & 21,9 & 15,5 & \multicolumn{2}{|c|}{38,7} & \multicolumn{2}{|c|}{38,7} & \multicolumn{2}{|c|}{39,2} & \multicolumn{2}{|c|}{145,5} & 34,0 & 34,0 \\
\hline 2 & 41,0 & 32,0 & \multicolumn{2}{|c|}{27,3} & \multicolumn{2}{|c|}{27,2} & \multicolumn{2}{|c|}{33,8} & 122,4 & 122,2 & 23,6 & 25,1 \\
\hline 3 & 214,5 & 72,1 & \multicolumn{2}{|c|}{80,6} & \multicolumn{2}{|c|}{79,6} & \multicolumn{2}{|c|}{219,9} & \multicolumn{2}{|c|}{119,5} & 75,0 & 75,0 \\
\hline 4 & 57,7 & 48,9 & \multicolumn{2}{|c|}{37,8} & & & \multicolumn{2}{|c|}{47,4} & \multicolumn{2}{|c|}{144,4} & 38,8 & 37,7 \\
\hline 5 & 41,7 & 37,6 & \multicolumn{2}{|c|}{55,2} & \multicolumn{2}{|c|}{55,1} & \multicolumn{2}{|c|}{55,2} & 115,7 & 114,9 & 49,5 & 49,5 \\
\hline 6 & 40,8 & 41,5 & \multicolumn{2}{|c|}{18,2} & & & \multicolumn{2}{|c|}{18,3} & 122,4 & 122,2 & 18,2 & 18,2 \\
\hline 7 & 18,2 & 17,8 & \multicolumn{2}{|c|}{32,8} & & & \multicolumn{2}{|c|}{32,5} & \multicolumn{2}{|c|}{25,7} & 34,7 & 34,8 \\
\hline 8 & 52,9 & 52,9 & \multicolumn{2}{|c|}{39,9} & & & \multicolumn{2}{|c|}{39,6} & 32,4 & 32,4 & 39,7 & 43,0 \\
\hline 9 & 37,0 & 33,4 & \multicolumn{2}{|c|}{47,5} & & & \multicolumn{2}{|c|}{46,8} & 74,4 & 74,6 & 50,5 & 56,0 \\
\hline 10 & 59,0 & 61,1 & & & & & & & 39,3 & $31,9^{\mathrm{c}}$ & 37,1 & 37,7 \\
\hline 11 & 35,6 & 35,2 & & & & & & & 24,6 & 24,5 & 21,0 & 21,0 \\
\hline 12 & 30,1 & 29,8 & 124,2 & 122,2 & 124,4 & 121,6 & 12,2 & 121,4 & $37,4^{\mathrm{c}}$ & $124,0^{\mathrm{c}}$ & 26,0 & 26,0 \\
\hline 13 & 37,4 & 38,0 & 140,0 & 146,0 & 139,6 & 145,2 & 13,7 & 145,2 & $37,7^{\mathrm{c}}$ & $135,2^{\mathrm{c}}$ & 38,0 & 38,0 \\
\hline 14 & 39,3 & 39,3 & & & 42,0 & 41,7 & 42,3 & 41,5 & 37,7 & 37,4 & 42,5 & 42,0 \\
\hline 15 & 32,4 & 30,5 & & & 28,7 & 26,1 & 29,1 & 26,8 & 24,5 & 25,7 & 27,2 & 26,8 \\
\hline 16 & 35,2 & 35,7 & & & 26,6 & 26.9 & 26,5 & 26,8 & $37,4^{\mathrm{c}}$ & $124,0^{\mathrm{c}}$ & 35,0 & 38,0 \\
\hline 17 & 29,6 & 30,1 & 33,6 & 32,7 & & & 34,1 & 32,4 & 37,8 & $135,2^{\mathrm{c}}$ & 43,5 & 34,8 \\
\hline 18 & 42,4 & 42,5 & 58,9 & 47,4 & 59,0 & 47,6 & 59,1 & 47,2 & 37,4 & $37,5^{\mathrm{c}}$ & 49,0 & 47,6 \\
\hline 19 & 34,9 & 35,0 & 39,9 & 47,1 & 39,6 & 46,7 & 39,6 & 46,7 & 24,8 & $25,7^{\mathrm{c}}$ & 47,6 & 39,7 \\
\hline 20 & 27,7 & 30,3 & 39,4 & 28,6 & 39,6 & 31,1 & 39,4 & 31,0 & $39,3^{\mathrm{c}}$ & $124,1^{\mathrm{c}}$ & 153,1 & 156,0 \\
\hline 21 & 32,0 & 32,5 & 32,7 & 33,6 & 31,2 & 34,7 & 31,2 & 34,8 & 27,9 & $131,0^{\mathrm{c}}$ & 29,4 & 25,6 \\
\hline 22 & 38,8 & 38,9 & 41,4 & 37,6 & 41,5 & 37,1 & 42,0 & 37,5 & $22,7^{\mathrm{c}}$ & 17,0 & 39,7 & 39,7 \\
\hline 23 & 6,0 & 11,3 & 28,0 & 28,0 & 28,0 & 28,4 & 28,0 & 28,4 & 22,7 & 26,0 & 28,0 & 28,0 \\
\hline 24 & 14,20 & 16,1 & 15,6 & 15,6 & 15,7 & 15,6 & 15,5 & 16,0 & 19,2 & 14,1 & 15,5 & 15,5 \\
\hline 25 & 17,50 & 17,9 & 15,6 & 15,6 & 15,7 & 15,7 & 16,0 & 15,7 & 19,2 & 14,1 & 16,0 & 16,7 \\
\hline 26 & 18,20 & 18,3 & 16,7 & 16,7 & 16,9 & 16,9 & 17,0 & 16,1 & 23,6 & 23,5 & 16,0 & 16,0 \\
\hline 27 & 19,80 & 19,7 & 23,1 & 25,8 & 23,3 & 26,1 & 23,5 & 26,3 & 11,7 & 11,8 & 14,1 & 14,1 \\
\hline 28 & 31,60 & 31,7 & 28,0 & 28,6 & 28,1 & 28,8 & 28,0 & 28,7 & 11,4 & 12,0 & 18,2 & 19,0 \\
\hline 29 & 34,50 & 34,7 & 33,1 & 33,6 & 23,3 & 33,4 & 23,6 & 33,5 & 11,6 & 11,6 & 109,1 & 106,1 \\
\hline 30 & 31.30 & 31,4 & 21,3 & 23,2 & 21,4 & 23,6 & 21,5 & 23,6 & & & 19,5 & 26,0 \\
\hline
\end{tabular}

${ }^{\mathrm{a}}$ Multiplicidade deduzida com base nos espectros PND e DEPT; ${ }^{\mathrm{b}} \mathrm{Os} \delta_{\mathrm{C}}$ da unidade cinamoila estão citados no texto; ${ }^{\mathrm{c}} \mathrm{O}$ espectro apresenta mais de um sinal de $\delta_{\mathrm{C}}$ próximo a este valor; ${ }^{\mathrm{d}}$ Numeração usada com o objetivo de facilitar a citação na tabela.

últimas frações da coluna continham material cristalino e, também, foram fracionadas através de cromatografia em coluna e camada preparativa usando como eluente diclorometano, acetato de etila e metanol em diferentes proporções. As frações foram então recristalizadas e os cristais analisados através de espectro de $\mathrm{RMN}{ }^{1} \mathrm{H}$ e ${ }^{13} \mathrm{C}$. Os constituintes foram identificados como uma mistura dos epímeros do lupeol e taraxasterol $(\mathbf{1 3 + 1 4}, 50.0 \mathrm{mg})$, fridelinol $\left(2 ; \mathrm{PF}: 360-62^{\circ} \mathrm{C} ; 130,0 \mathrm{mg}\right)$ e a mistura de cinamato de $\alpha$ - $+\beta$-amirina $\left(3+4\right.$; $\mathrm{PF}$ : $171-73^{\circ} \mathrm{C}$; $130,0 \mathrm{mg}$ ).

O extrato hexânico das folhas $(1,83 \mathrm{~g})$ foi fracionado em coluna de gel de sílica, usando-se diclorometano como eluente inicial e aumentando-se a polariadade com acetato de etila e metanol até metanol puro. Recolheram-se 181 frações de 10 $\mathrm{ml}$ cada uma que foram analisadas através de cromatografia em camada fina e reunidas em 10 grupos de frações. Estas foram submetidas a processos de purificação através de cromatografia em camada preparativa normal e/ou centrífuga e cristalização. As frações foram analisadas através de espectros IV e RMN ${ }^{1} \mathrm{H}$ e ${ }^{13} \mathrm{C}$. Destes processos foram identificadas misturas de hidrocarbonetos alifáticos, álcoois alifáticos, a mistura dos triterpenos $\alpha-+\beta$-amirina $\left(5+6\right.$; PF: $171-173^{\circ} \mathrm{C}$, $260,0 \mathrm{mg}$ ) e a mistura de $\alpha-+\beta$-amirenona $(7+8$; PF: 101 $\left.103^{\circ} \mathrm{C}, 136,0 \mathrm{mg}\right)$.

\section{AGRADECIMENTOS}

Os autores agradecem ao CNPq, CAPES pela concessão de auxílio financeiro e de bolsas (Iniciação Científica, PósGraduação e Pesquisa) e a FAPERJ e PADCT/FINEP pelos auxílios concedidos.

\section{REFERÊNCIAS}

1. Brito, N. R. S. de; Tese de Doutoramento, Intituto de Química-USP, São Paulo, 1985.

2. Pant, P.; Rastogi, R. P.; Phytochemistry 1979, 18, 1095.

3. Das, M. L.; Mahato, S. B.; Phytochemistry 1983, 22,1071

4. Pal, B. C.; Achari, B.; Price, K. R.; Phytochemistry 1991, 30,4177

5. Massiot, G.; Chen, X.; Lavand, C.; LeMen-Olivier, L.; Delando, C.; Viari, A.; Vigny, P.; Duval, J.; Phytochemistry 1992, 31, 3571.

6. Bergmlan, J.; Phytochemistry 1984, 18, 3547.

7. a. Mello, N. L. de; Carvalho, A. M. de; Cháves, J. P.; David, J. M.; Cruz, F. G.; Resumos-SBQ 1994, PN-036; b. Carvalho, M. G. de; Hauptli, M. B.; Almeida, M. E. L. de; Resumos-SBQ 1991, PN-030; c. Carvalho, M. G. de; Bezerra, F. B.; Resumos-SBQ 1993, PN-016.

8. Carvalho, M. G. de; Almeida, M. E. L. de; Halptli, M. B.; Meleiro, L. A. C.; Rev. Univ. Rural, Sér. Ciên. Exatas e da Terra, 1995, jan./dez. 17(1/2), 33.

9. Olea, R. S. G.; Roque, N. F.; Quím. Nova 1990, 13, 278.

10. Patra, A.; Mukhopadhyay, A. K.; Mitra, A. K.; Org. Magn. Res. 1981, 17, 165.

11. Mahato, S. B.; Kundu, A. P.; Phytochemistry 1994, 37, 1517.

12. White, D. E.; Pure and Applied Chemistry; 1956, 6, 191.

13. Aragão, P. C. de A.; Toledo, J. B. de; Morais, A. A.; Braz-Filho, R.; Quím. Nova 1990, 13, 254.

14. Patra, A.; Chaudhuri, S. K.; Ruegger, H. J.; J. Indian Chem. Soc. 1990, 67, 394. 
15. Oksuz, S.; Topcu, G.; Phytochemistry 1987, 26, 3082. 16. Seo, S.; Tomita, Y. Tori, K.; Tetrahedron Lett. 1975, 7. 17. Knighst, S. A.; Org. Magn. Res. 1974, 6, 603.

18. Bhattacharyya, J.; Barros, C. B.; Phytochemistry 1986, $25,274$.

19. Delgado, M. C. C.; Silva, M. S. da; Braz-Filho, R.; Quím. Nova 1986, 9, 119.

20. Buckingman, V.; Dictionary of Natural Products Chapman \& Hall: 2-6, London, 1994.

21. Marner, F.; Frayer, A.; Lex, J.; Phytochemistry 1991, 30,3709
22. Bolzeni, V. da S.; Trevisan, L. M. V.; Young, M. C. M.; Phytochemistry 1991, 30, 2089.

23. Goulart, M. O. F.; Sant'Ana, A. E. G.; Lima, R. A. de; Cavalcante, S. H.; Carvalho, M. G. de; Braz-Filho, R.; Quím. Nova 1993, 16, 95.

24. Bhacca, N. S.; Johnson, L. F.; Shoolery, J. N. S.; High Resolution NMR Spectra Catalog, of the analytical Instr. Div. of Varian, Nat. Pres., USA(1962), 366.

25. Matsuo, M., Urano, S.; Tetrahedron 1975, 32, 229.

26. Singh, B.; Agrawal, P. K.; Thakur, R.; Phytochemistry 1989, 28, 1980. 